Journal of

Synchrotron

Radiation

ISSN 0909-0495

Received 10 March 2005

Accepted 17 June 2005

C) 2005 International Union of Crystallography

Printed in Great Britain - all rights reserved

\section{X-ray excited visible luminescence spectroscopy of organic materials using a portable optical spectrometer}

\author{
Vincenzo Maiorano, ${ }^{a}$ Francesca Matino, ${ }^{a}$ Roberto Cingolani, ${ }^{a}$ Julie Thompson ${ }^{\mathrm{a}, \mathrm{b}}$ \\ and Robert I. R. Blyth ${ }^{\mathrm{a} *}$ \\ ${ }^{a}$ National Nanotechnology Laboratory of INFM, Distretto Technologico della Università di Lecce, \\ via Arnesano, 73100 Lecce, Italy, and ${ }^{\mathbf{b}}$ Float-Lux srl, via Ravenna 14, 73100 Lecce, Italy. \\ E-mail: rob.blyth@unile.it
}

The use of a portable video telescope, mounted externally to a beamline endstation, to obtain synchrotron-radiation-excited visible luminescence, is described. Real-time video monitoring permits simple and quick alignment, and allows a visual record of the luminescence experiment. The telescope is fibreoptic-coupled to an optical spectrometer. Examples are given of X-ray excited optical spectroscopy from organic materials for light-emitting-diode applications.

Keywords: visible luminescence; optical spectroscopy; organic molecules.

\section{Introduction}

Optical spectroscopy of materials is a standard technique, giving particular insights into processes on the typical energy scales used in electronic devices, and being of obvious use when it is the visible light-emitting properties of the materials themselves which are of interest. Synchrotron radiation is a valuable addition to the range of possible excitation sources (Sham et al., 1993, 2001; Rogalev \& Goulon, 2001; Hu et al., 2002), with the intrinsic tunability giving the opportunity to study site-specific exciton formation by using photon energies at the appropriate absorption edge. This technique is generally known by the acronym XEOL (X-ray excited optical luminescence), although this term by implication also includes excitation by fixed anode sources.

There are several methodologies for the detection of synchrotron-radiation-excited visible luminescence. The first route is to have a dedicated beamline and luminescence spectrometer, such as the Superlumi beamline at HASYLAB. The second is to have a dedicated mobile endstation, able to be used on multiple beamlines, such as the MoLES endstation at Daresbury (Quinn et al., 2003). The third is to use an existing beamline and endstation and mount an optical detection system externally, using the chamber viewports, as was done for some of the early studies (e.g. Bianconi et al., 1978). From the perspective of a fully optimized detection system, clearly the dedicated beamline and/or endstation is the preferred route. The problems with externally mounted optical detection systems are twofold: firstly, the fraction of the emitted light collected is very limited, since the closest a lens can be placed to the sample is limited by the chamber size.
Note that a partial solution, having a single lens inside the chamber and the rest of the collection optics outside, has also been used. The second problem is that of alignment. Collection of light from a sample and focusing it on the entrance slit of a spectrometer requires multiple lenses. The alignment of these lenses, constrained by the geometry of the chamber, is in general a non-trivial exercise. Further, this is likely to be required to be performed under time pressure (beam time being a valuable commodity). Finally, it is likely also to be performed in a well lit environment, quite unlike the dark conditions in dedicated optical spectroscopy laboratories. For these reasons, most synchrotron-radiation-excited optical experiments are performed on dedicated systems.

In this work we demonstrate a simple, externally mounted, fully portable optical detection system that can be aligned in moments. As examples we show data from a class of materials where the technological interest lies in their light-emitting properties: luminescent organic molecules. The interest in such materials has grown enormously in recent years, fuelled by developments in organic light-emitting diode (LED) technology, and they have already been the subject of XEOL studies (Sham et al., 2000; Naftel et al., 2001, 2003; Hu et al., 2002). The materials used here were aluminium tris-8hydroxyquinoline, $\mathrm{AlQ}_{3}$, the polyfluorene polymer poly[(9,9dioctylfluorenylene-2,7-diyl) - co-(1,4-diphenylene-vinylene-2methoxy-5-\{2-ethylhexyloxy\}-benzene)], which for convenience we will refer to by its commercial name ADS125GE, and the iridium complexes iridium bis[2-(4,6-difluorophenyl)pyridinato- $\left.N, C^{2 \prime}\right]$ picolinate, FIrpic, and iridium bis[2(2'-benzothienyl)pyridinato- $\left.N, C^{3 \prime}\right]$ (acetylacetonate), btpy2$\operatorname{Ir}(\mathrm{acac})$. These last two molecules, which suffer from 
concentration quenching of their luminescence, were doped into a polymer host, poly-9-vinylcarbazole, PVK. The structures of all these molecules are shown in Fig. 1.

\section{Equipment and samples}

\subsection{Beamline}

The measurements were performed on the BEAR beamline (Nannarone et al., 2004) at the ELETTRA synchrotron radiation facility, Trieste, Italy. This is a bending-magnet beamline, covering the photon energy range $5-1600 \mathrm{eV}$, while still having a significant visible contribution to zero order. The BEAR endstation has two chambers. The experimental chamber is located such that the sample is at a distance of $150 \mathrm{~cm}$ from the refocusing mirror, at which point the light is focused, giving a typical spot size of $30 \mu \mathrm{m} \times 100 \mu \mathrm{m}$. For organic molecules, it is known that high densities of X-rays can cause material damage (Treusch et al., 1999; Coffey et al., 2002), so for both optical and (drain current) NEXAFS measurements, which are not limited by the focal requirements of an electron spectrometer, we used the BEAR preparation chamber. This chamber is located directly downstream of the experimental chamber, with the sample now at $\sim 240 \mathrm{~cm}$ from the refocusing mirror. Here the beam is of the order of a few $\mathrm{cm}$ in size, reducing the flux density by five orders of magnitude. The beam size was cut using baffles, such that it was the same size as the samples. In this way NEXAFS spectra could be recorded using drain current measurements,<smiles>Oc1cccc2cccnc12</smiles><smiles>CC(C)C(C)(C)Cn1c2ccccc2c2ccccc21</smiles>

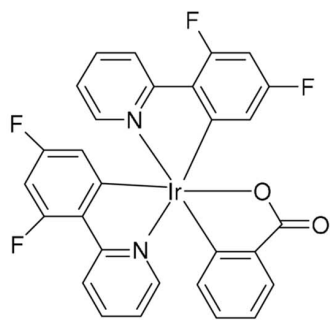

FIrpic

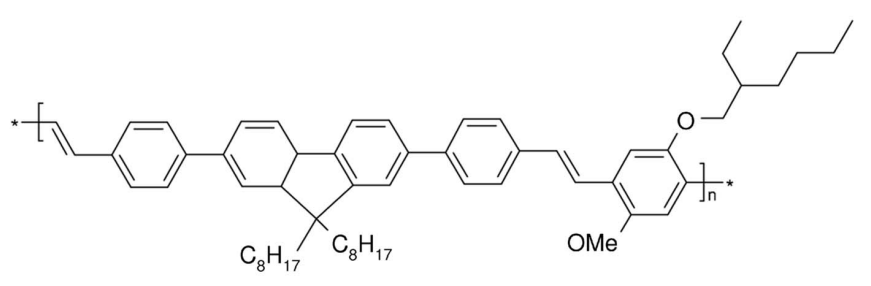

Figure 1

ADS125GE

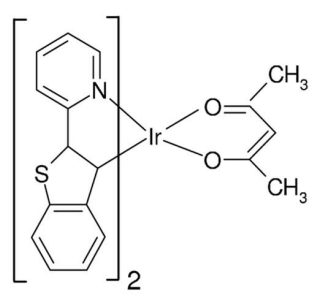

btpy2Ir(acac)

Molecular structures of the organic materials studied. without a contribution from any adventitious contamination on the sample holder.

\subsection{Video telescope}

The video telescope used was the Optronics OL610. This unit is designed to be portable, weighing $1.1 \mathrm{~kg}$, and with dimensions of $13 \mathrm{~cm} \times 12 \mathrm{~cm} \times 7.5 \mathrm{~cm}$, with an additional $10 \mathrm{~cm}$ taken up by the lens. The internal layout of the video telescope is shown in Fig. 2.

The objective lens has a focusing range from $7 \mathrm{~cm}$ to infinity, allowing the unit to be placed directly adjacent to viewports on most UHV chambers. The CCD video camera images the mirror immediately behind the objective lens, while a portion of the optical signal passes through an aperture and is fed via a system of transfer lenses to a fibre-optic coupling. This can then be sent via fibre optic to an optical spectrometer. The aperture size determines the effective field of view of the optical spectrometer. The field-of-view aperture appears as a black spot on the video image, with all signal from that portion of the image being sent to the spectrometer. This makes alignment of the video telescope particularly easy, as the video image can be monitored in real time, with the black dot on the image simply placed over the area from which the visible signal is anticipated. This is illustrated in Fig. 3, which shows a

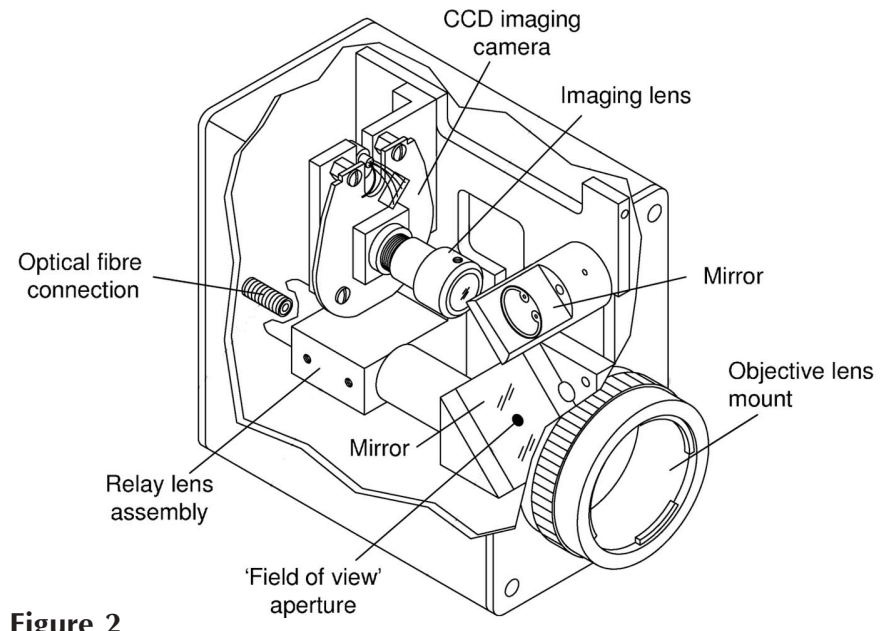

Internal layout of the Optronics OL610 video telescope.

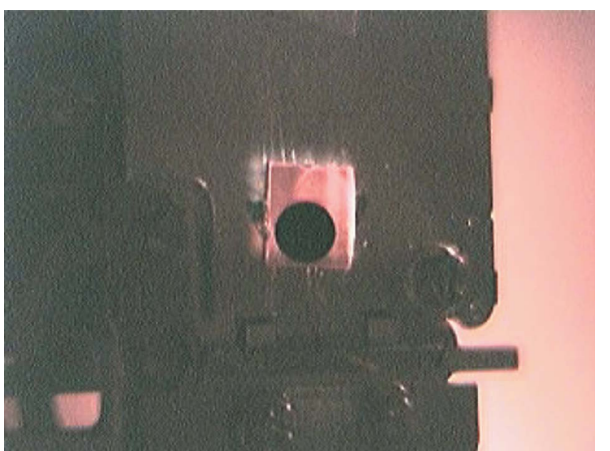

Figure 3

Video capture of the sample set-up, with a sample illuminated by zeroorder radiation, and the ion gauge on. 
video image captured during alignment on BEAR. The image was recorded with the ion gauge on, giving sufficient illumination for alignment. The image shows the BEAR sample holder (VG XL25), with a luminescent organic film on a $\sim 1 \mathrm{~cm} \times 1 \mathrm{~cm}$ substrate. The beamline is set to zero order. The beam size at the sample is very slightly larger than the sample in this case, as can be seen by the white light at the top right of the sample, which is itself fluorescing a red colour. The video telescope itself has a standard camera mount in its underside, and this was used to attach it to a laboratory jack, which was placed near a BEAR preparation chamber viewport, a standard UHV viewport, i.e. not a specialized component. The transmission of standard glass is extremely flat in the range $400-1000 \mathrm{~nm}$, the range used in this work. Note however, that it falls very rapidly below $400 \mathrm{~nm}$, which must be taken into account during calibration. For such low wavelengths, quartz windows are to be preferred.

The distance from the objective lens to the sample was $35 \mathrm{~cm}$. Alignment was performed simply by hand, no precision alignment being required. Aluminium foil taped to the unit and to the chamber was sufficient to block all ambient light (all other viewports being foil-covered) while remaining flexible enough to allow adjustments of the alignment. As can be seen from Fig. 3, the size of the field of view was such that it enabled simultaneous capture of an image of part of the sample under illumination with the recording of the optical spectrum.

\subsection{Spectrometer}

The spectrometer used was the Optronics OL770 unit, designed and marketed for LED characterization. The input is fibre-optic coupled to the OL610 telescope unit. The spectrometer has a fixed single grating, with the detector being a two-dimensional CCD. The use of a fixed-grating CCD spectrometer allows the recording of all wavelengths in the optical spectrum simultaneously, considerably reducing the acquisition time. The CCD is thermoelectrically cooled to $263 \mathrm{~K}$, giving a quantum efficiency of $>90 \%$ at a wavelength of $650 \mathrm{~nm}$. The entrance/exit slits are fixed at $100 \mu \mathrm{m}$, giving a wavelength resolution of $0.4 \mathrm{~nm}$, with a wavelength range of $380-780 \mathrm{~nm}$, i.e. strictly limited to the visible part of the electromagnetic spectrum. The spectral response of the entire system, telescope, fibres, grating and detector, was thoroughly calibrated to industrial standards by the manufacturer. Note that, in the case of using a non-commercial set-up, careful calibration must be performed in order to take into account the non-ideal spectral response of each component.

The unit is designed for portability, and has a weight of $10.2 \mathrm{~kg}$, with dimensions of $18 \mathrm{~cm} \times 34 \mathrm{~cm} \times 33 \mathrm{~cm}$. The computer interface was via USB cable, allowing use with laptop computers, as was the case for this experiment.

\subsection{Organic sample preparation}

ADS125GE, FIrpic and btpy2Ir(acac) were obtained from American Dye Source, Quebec, Canada, while $\mathrm{AlQ}_{3}$ and PVK were obtained from Sigma-Aldrich. All materials were used as received. $\mathrm{AlQ}_{3}$ films, approximately $70 \mathrm{~nm}$ thick, were evaporated onto glass and copper substrates under high vacuum $\left(10^{-4} \mathrm{~Pa}\right)$ at evaporation rates of $0.2-0.3 \AA \mathrm{s}^{-1}$, determined by a quartz crystal microbalance. Samples of the ADS125GE polymer and the PVK/Ir complex blends were prepared by drop-coating from solution, giving films of unknown thickness. All samples were inserted into the vacuum chamber using a fast-entry lock.

\section{Examples of X-ray excited optical luminescence from organic materials}

In order to give a true idea of the data quality possible, no image processing or data smoothing has been applied to any of the following data. X-ray excited optical luminescence of organic materials differs fundamentally from conventional UV or visible-excited photoluminescence. In the latter case, the exciton is generated directly by the optical excitation of an electron into the conduction band leaving a hole in the valence band. Since the ground state of an organic molecule is a singlet, this optical excitation produces a singlet excited state. In X-ray excited luminescence the electron is again placed directly in the conduction band, assuming the photon energy is tuned to, or close to, an absorption edge, or has a significant content at such energies, e.g. zero-order light. However, here the hole in the valence band arrives via the decay of the core hole, a multi-electron process which permits the hole to have a different spin to that obtained via direct optical excitation, i.e. $\mathrm{X}$-ray excited luminescence is a more efficient producer of triplet excitons. This mimics the spin statistics in organic LEDs where, under electrical excitation, $75 \%$ of the generated excitons are triplets.

The phosphorescent iridium complexes developed by Forrest and co-workers (Baldo et al., 2000) have been used to make extremely efficient organic LEDs. This is due to the fact that the luminescence is derived from triplet states, which form a majority of excitons in an electrically excited device such as an LED. Recently, near $100 \%$ internal concentration has been demonstrated in an iridium-complex-based LED (Adachi et al., 2001). These molecules suffer from concentration quenching of their luminescence, and for device purposes are blended with a suitable host material. Fig. 4 shows X-ray excited luminescence spectra of the blue emitter FIrpic, and the red emitter btpy2Ir(acac), each in a PVK host. This polymer/Ir complex combination has been recently used to produce a white-emitting organic LED (Thompson et al., 2005; Maiorano et al., 2005). The excitation energy was $286 \mathrm{eV}$, which corresponds to absorption from the $\mathrm{C} 1 s$ levels to the lowest unoccupied orbital of the PVK host, as can be seen from the NEXAFS spectrum of PVK in Fig. 5.

The spectrum from btpy $2 \operatorname{Ir}(\mathrm{acac})$ has a peak at $620 \mathrm{~nm}$, with two clear vibrational replicas. The spectrum from FIrpic is peaked around $500 \mathrm{~nm}$, but is more complex, with four components evident from a visual inspection. It is generally considered that emission is seen from two distinct triplet excited states in this family of molecules: the metal-ligand charge-transfer state ${ }^{3}$ MLCT, and the $\pi-\pi^{*}$ exciton. The $\pi-\pi^{*}$ emission is often distinguished by a relatively broad peak with 


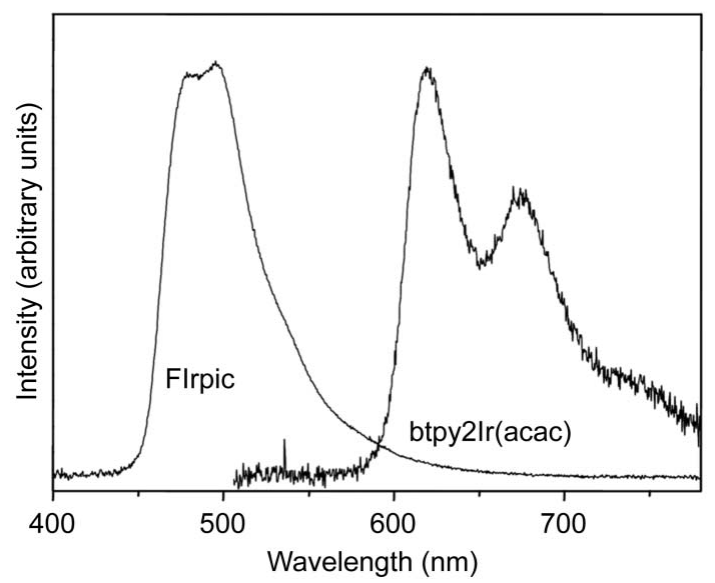

Figure 4

Visible luminescence spectra of films of the iridium complexes FIrpic and btpy $2 \operatorname{Ir}(\mathrm{acac})$ in a PVK polymer host. The excitation energy was $286 \mathrm{eV}$, and the total acquisition time was 10 min per spectrum.

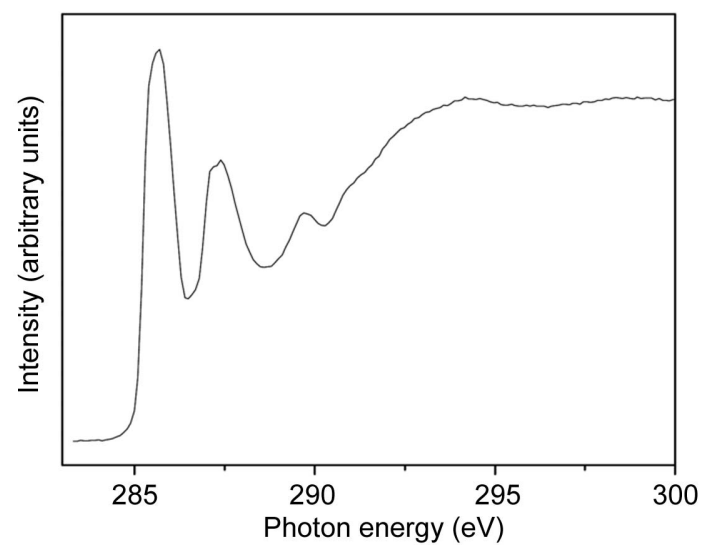

Figure 5

Carbon $K$-edge NEXAFS spectrum of the polymer PVK.

vibronic replicas, and a large Stokes shift from the ${ }^{3} \mathrm{MLCT}$ absorption peak (Lamansky et al., 2001), whereas the ${ }^{3}$ MLCT peak is usually at a higher energy, without any vibronic replicas. A curve fit to the FIrpic spectrum is shown in Fig. 6. Note that this fit is performed using an energy scale rather than wavelength, since vibrational replicas are at a constant energy separation. Initial results implied a single narrow peak at the high-energy side $(A)$, with three broader peaks at lower energies $(B-D)$. Assuming that the three broad peaks consisted of a $\pi-\pi^{*}$ emission plus two vibrational replicas, the fit was re-performed constraining the widths of peaks $B-D$ to be the same. The results of this fit are shown in Fig. 6. The good quality of this fit suggests that peak $A$ is a ${ }^{3}$ MLCT peak, while peaks $B-D$ are indeed a $\pi-\pi^{*}$ peak plus vibrational replicas. It is these vibrational replicas which give a sufficiently high green component to the emitted light from a combined PVK/FIrpic + btpy2Ir(acac) LED for the overall emission to be white (Thompson et al., 2005; Maiorano et al., 2005).

The high-intensity signals possible using phosphorescent organics are illustrated in Fig. 7. This shows a spectrum of the $\mathrm{PVK} / \mathrm{btpy} 2 \operatorname{Ir}(\mathrm{acac})$ sample under zero-order illumination. The

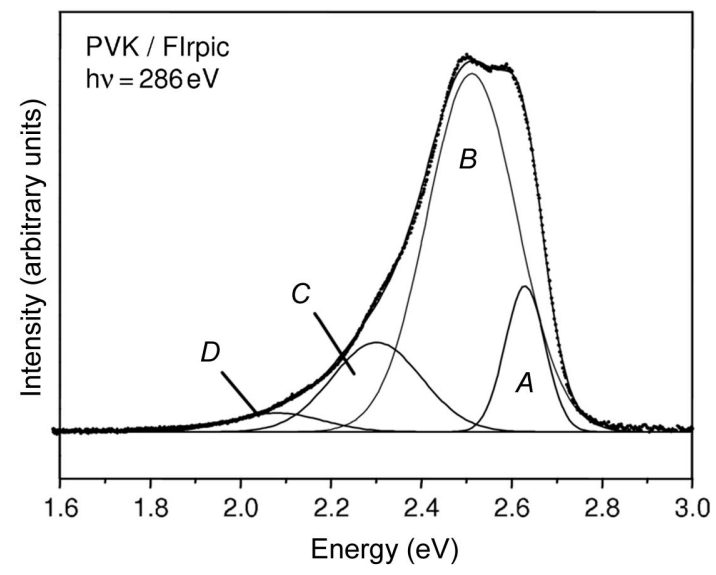

Figure 6

Curve fit to the FIrpic spectrum of Fig. 4. Note that this spectrum is on an energy scale.

spectrum shows excellent statistics, even though the total acquisition time was only $100 \mathrm{~ms}$. Note the rising background at the low-wavelength region of the spectrum. This is the visible component of zero order, which is the familiar bluewhite, i.e. has higher intensity at low wavelengths. The inset shows the video capture acquired simultaneously with the spectrum. The sample appears white, not because the sample is luminescing white light but because the intensity of the signal has overloaded the CCD camera. That the film is actually emitting red light is demonstrated by the red signal immediately surrounding the sample, which is due to reflected red emission from the edge of the organic film.

A beamline such as BEAR, which has a window valve downstream of the monochromator, allows the use of just the visible component of zero-order light as the excitation source. This mimics more closely the conditions of conventional photoluminescence, since the enhanced triplet production via core-level excitation is largely avoided, assuming that the transmission of the window to X-rays is negligible. Fig. 8 shows such a photoluminescence spectrum of the polyfluorene polymer ADS125GE. Note again the excellent statistics despite the low total data-acquisition time, and again the rising background at low wavelengths, owing to the visible zeroorder light itself. The inset shows the simultaneous video capture, the emission being an evident, though relatively lowintensity, green colour.

The ability to acquire video images not only allows a visual record of the experiment, as in Figs. 7 and 8, but also, in certain cases, allows spectroscopic interpretation to be applied directly to the images. Fig. 9 shows video captures of samples under full zero-order illumination, i.e. with the window valve open. $\mathrm{AlQ}_{3}$ was the subject of the first reported organic LED (van Tang \& Slyke, 1987), and still remains important in the field. It is well known as a green-emitting material, having an emission maximum at around $530 \mathrm{~nm}$ (van Tang \& Slyke, 1987). The emission from the $\mathrm{AlQ}_{3}$ film on glass in Fig. 9 can, indeed, be seen to be green. Metallic $\mathrm{Cu}$ fluorescence emission has a maximum at around $600 \mathrm{~nm}$ (Berndt et al., 1991), and therefore appears red. The image of a $\mathrm{Cu}$ sample in Fig. 9 


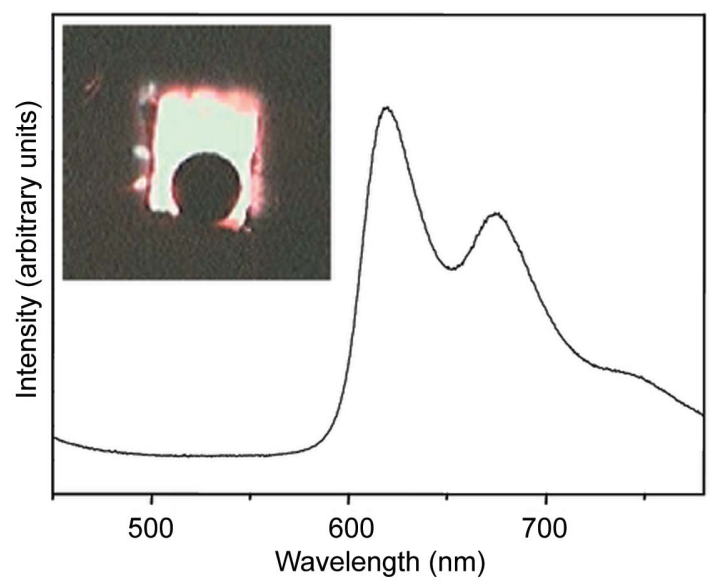

Figure 7

Visible luminescence spectrum of a film of PVK/btpy2Ir(acac) under full zero-order excitation. The total acquisition time was $100 \mathrm{~ms}$. The inset shows the simultaneously recorded video image.

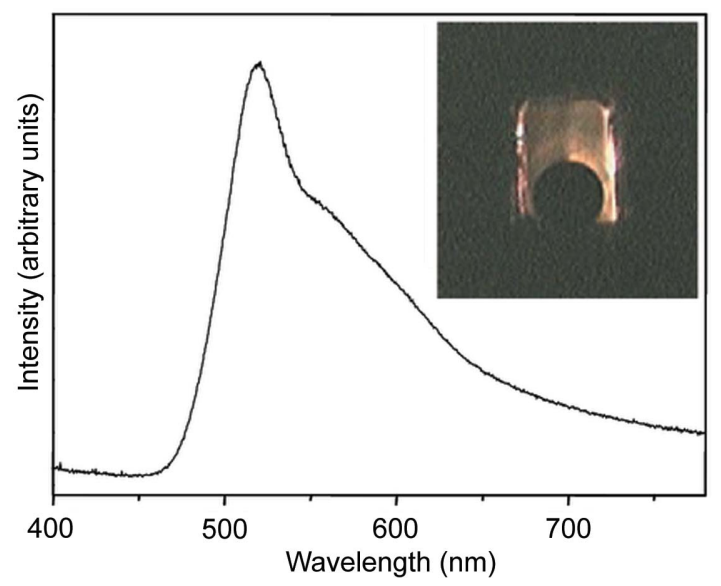

Figure 8

Visible luminescence spectrum of the polyfluorene ADS125GE. The excitation is the visible part of the zero-order spectrum only. The total acquisition time was $1 \mathrm{~min}$. The inset shows the simultaneously recorded video image.

shows no particular emission colour; the white outline is from the zero-order light hitting the less polished (and thus more diffusely reflective) sample holder. The low intensity from the $\mathrm{Cu}$ sample is due to the low quantum efficiency of the $\mathrm{Cu}$ fluorescence emission (Berndt et al., 1991). However, the image of the $\mathrm{AlQ}_{3}$ film on $\mathrm{Cu}$ shows clear red emission, rather than green. This shows that there is a coupling between the $\mathrm{AlQ}_{3}$ exciton and the excitation of the $\mathrm{Cu}$ substrate, which leads to greatly enhanced $\mathrm{Cu}$ emission rather than emission from the organic itself. This is not seen in conventional photoluminescence (Matino et al., 2005), but is seen in tunneling luminescence of organics on metals (Fujita et al., 2000). This suggests that the generation of triplets may be responsible for the enhanced metallic emission, since both $\mathrm{X}$-ray excited luminescence and tunneling spectroscopy generate triplet states, whereas UV photoluminescence does not.
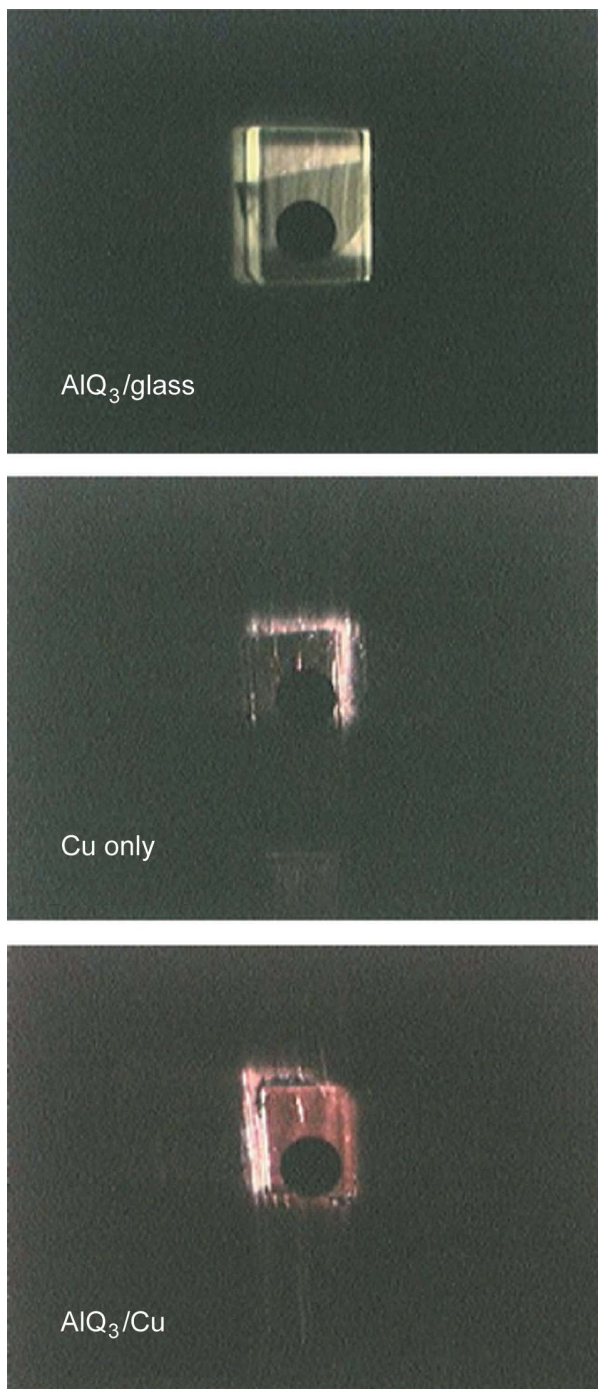

Figure 9

Video-capture images of three samples under full zero-order excitation.

\section{Conclusions}

We have shown that a simple, externally mounted, video telescope system can be easily aligned, and used to obtain visible luminescence spectra under synchrotron radiation excitation. This allows the addition of this spectroscopy to beamlines that are not dedicated to luminescence, without the need for a dedicated endstation, while avoiding the set-up and alignment problems inherent in a multiple-lens assembly.

For organic materials, this technique offers several advantages. The use of X-ray excitation, rather than UV or visible excitation, for luminescence spectroscopy more closely mimics the spin statistics of LEDs under electrical excitation. Further, the increased triplet production gives high signals from phosphorescent organics, i.e. exactly those materials which have produced record-setting efficiencies in the field. In addition, the system can be used to collect conventional photoluminescence spectra using the visible component of zero-order light. While it might at first seem superfluous to acquire photoluminescence spectra using a synchrotron when 
conventional laboratory sources such as lamps and lasers are readily available, what it does give is the ability of the synchrotron experimentalist to acquire photoluminescence spectra in situ. Note that this could also be achieved using a laser mounted on the experimental chamber, although lasers for organic photoluminescence need to operate in the near $\mathrm{UV}$, which raises both financial and safety issues. This in situ technique gives not only complementary data to that acquired with conventional synchrotron techniques, on the same sample in the same chamber, but also gives a point of reference for those less familiar with synchrotron spectroscopies. There is a large body of work, particularly photoemission and NEXAFS studies, on luminescent organic materials, but its penetration into the field of organic LED fabrication has been minimal. To include luminescence spectra in such studies would serve to control that the fundamental luminescent properties of the organic, i.e. those of most interest to the LED field, are unaffected by the unusual conditions of a (particularly a UHV) synchrotron experiment. It would also give some common ground between the synchrotron spectroscopist and the LED community.

The OL770 spectrometer, being designed for LED characterization, is not optimized for low signals. In particular, the thermal noise of the CCD is of the order of a few tens of counts per second per pixel, which is of the order of the signals to be expected from less efficient emitters. The use of a more highly cooled CCD spectrometer, where thermal noise is essentially zero, would open up a larger range of materials to study. The system also has a number of features directly related to LED characterization which are not relevant to spectroscopy, making it a sub-optimal, and simultaneously not a low-cost, option. The fibre optic output of the video telescope, however, does permit the use of any other fibre-opticcoupled optical spectrometer, of which there are any number of low-cost options on the market. Standard frame-grabbing software can be used to retain the video capture capability without the OL770 unit.

Further, the OL770 does not have an output permitting the acquisition of the total signal strength, which could be used to monitor the emission intensity as a function of photon energy. The use of a spectrometer which does have such an output would permit the acquisition of NEXAFS spectra, as has been demonstrated for organics by Sham and co-workers (Sham et al., 2000; Naftel et al., 2001, 2003; Hu et al., 2002). This would permit not simply a further option for NEXAFS detection but also the acquisition of NEXAFS spectra from samples on insulating substrates. Given that the field of organic electronic devices, such as transistors and diodes, requires the study of such molecules on insulating substrates, this may well be an important tool.
We thank the BEAR staff, particularly Nicola Mahne, for assistance at ELETTRA, Optronics for permission to reproduce the layout of the OL610, and Sonia Carallo and Betty Perrone for technical assistance at the NNL.

\section{References}

Adachi, C., Baldo, M. A., Thompson, M. E. \& Forrest, S. R. (2001). J. Appl. Phys. 90, 5048-5051.

Baldo, M. A., Thompson, M. E. \& Forrest, S. R. (2000). Nature (London), 403, 750-753.

Berndt, R., Gimzewski, J. K. \& Johansson, P. (1991). Phys. Rev. Lett. 67, 3796-3799.

Bianconi, A., Jackson, D. \& Monahan, K. (1978). Phys. Rev. B, 17, 2021-2024.

Coffey, T., Urquhart, S. G. \& Ade, H. (2002). J. Electron. Spectrosc. Relat. Phenom. 122, 65-78.

Fujita, D., Ohgi, T., Deng, W.-L., Nejo, H., Okamoto, T., Yokoyama, S., Kamikado, K. \& Miahiko, S. (2000). Surf. Sci. 454, 1021-1025.

Hu, Y. F., Tan, K. H., Kim, P.-S., Zhang, P., Naftel, S. J., Sham, T. K., Coulthard, I. \& Yates, B. W. (2002). Rev. Sci. Instrum. 73, 13791381.

Lamansky, S., Djurovich, P., Murphy, D., Abdel-Razzaq, F., Lee, H.-E., Adachi, C., Burrows, P. E., Forrest, S. R. \& Thompson, M. E. (2001). J. Am. Chem. Soc. 123, 4304-4312.

Maiorano, V., Perrone, E., Carallo, S., Cingolani, R., Blyth, R. I. R. \& Thompson, J. (2005). Synth. Met. In the press.

Matino, F., Maiorano, V., Cingolani, R., Thompson, J. \& Blyth, R. I. R. (2005). To be published.

Naftel, S. J., Kim, P.-S., Sham, T. K., Sammynaiken, R., Yates, B. W. \& Hu, Y. F. (2003). J. Appl. Phys. 93, 5191-5195.

Naftel, S. J., Zhang, P., Kim, P.-S., Sham, T. K., Coulthard, I., Antel, W. J. Jr, Freeland, J. W., Frigo, S. P., Fung, M. K., Lee, S. T., Hu, Y.-F. \& Yates, B. W. (2001). Appl. Phys. Lett. 78, 1847-1849.

Nannarone, S., Borgatti, F., DeLuisa, A., Doyle, B. P., Gazzadi, G. C., Giglia, A., Finetti, P., Mahne, N., Pasquali, L., Pedio, M., Selvaggi, G., Naletto, G., Pelizzo, M. G. \& Tondello, G. (2004). AIP Conf. Proc. 705, 450-453.

Quinn, F., Poolton, N., Malins, A., Pantos, E., Andersen, C., Denby, P., Dhanak, V. \& Miller, G. (2003). J. Synchrotron. Rad. 10, 461-466.

Rogalev, A. \& Goulon, J. (2001). In Chemical Applications of Synchrotron Radiation, edited by T. K. Sham. Singapore: World Scientific.

Sham, T. K., Jiang, D. T., Coulthard, I., Lorimer, J. W., Feng, X. H., Tan, K. H., Frigo, S. P., Rosenberg, R. A., Houghton, D. C. \& Bryskiewicz, B. (1993). Nature (London), 363, 331-334.

Sham, T. K., Naftel, S. J. \& Coulthard, I. (2001). In Chemical Applications of Synchrotron Radiation, edited by T. K. Sham. Singapore: World Scientific.

Sham, T. K., Sammynaiken, R., Zhu, Y. J., Zhang, P., Coulthard, I. \& Naftel, S. J. (2000). Thin Solid Films, 363, 318-321.

Tang, C. W. \& van Slyke, S. A. (1987). Appl. Phys. Lett. 51, 913-915.

Thompson, J., Maiorano, V., Carallo, S., Perrone, E., Biasco, A., Cingolani, R., Croce, A., Daneu, A. \& Blyth, R. I. R. (2005). Synth. Met. In the press.

Treusch, R., Himpsel, F. J., Kakar, S., Terminello, L. J., Heske, C., van Buuren, T., Dinh, V. V., Lee, H. W., Pakbaz, K., Fox, G. \& Jimenez, I. (1999). J. Appl. Phys. 86, 88-93. 